\title{
The crystal structure of the catalytic domain of a eukaryotic guanylate cyclase Jonathan A Winger ${ }^{1}$, Emily R Derbyshire ${ }^{1}$, Meindert H Lamers ${ }^{1}$, Michael A Marletta1,2,4 and John Kuriyan*1,2,3
}

Address: ${ }^{1}$ Department of Molecular and Cell Biology, University of California, Berkeley, CA, USA, ${ }^{2}$ Department of Chemistry, University of California, Berkeley, CA, USA, ${ }^{3}$ Howard Hughes Medical Institute, University of California, Berkeley, CA, USA and ${ }^{4}$ Division of Physical Biosciences, Lawrence Berkeley National Lab, Berkeley, CA, USA

Email: Jonathan A Winger - wingerj@berkeley.edu; Emily R Derbyshire - derbyem@berkeley.edu; Meindert H Lamers - mlamers@berkeley.edu; Michael A Marletta - marletta@berkeley.edu; John Kuriyan* - kuriyan@berkeley.edu

* Corresponding author

Published: 7 October 2008

BMC Structural Biology 2008, 8:42 doi:10.1 I86/1472-6807-8-42

This article is available from: http://www.biomedcentral.com/1472-6807/8/42

(C) 2008 Winger et al; licensee BioMed Central Ltd.

This is an Open Access article distributed under the terms of the Creative Commons Attribution License (http://creativecommons.org/licenses/by/2.0), which permits unrestricted use, distribution, and reproduction in any medium, provided the original work is properly cited.
Received: 17 September 2008

Accepted: 7 October 2008

\begin{abstract}
Background: Soluble guanylate cyclases generate cyclic GMP when bound to nitric oxide, thereby linking nitric oxide levels to the control of processes such as vascular homeostasis and neurotransmission. The guanylate cyclase catalytic module, for which no structure has been determined at present, is a class III nucleotide cyclase domain that is also found in mammalian membrane-bound guanylate and adenylate cyclases.

Results: We have determined the crystal structure of the catalytic domain of a soluble guanylate cyclase from the green algae Chlamydomonas reinhardtii at $2.55 \AA$ resolution, and show that it is a dimeric molecule.

Conclusion: Comparison of the structure of the guanylate cyclase domain with the known structures of adenylate cyclases confirms the close similarity in architecture between these two enzymes, as expected from their sequence similarity. The comparison also suggests that the crystallized guanylate cyclase is in an inactive conformation, and the structure provides indications as to how activation might occur. We demonstrate that the two active sites in the dimer exhibit positive cooperativity, with a Hill coefficient of $\sim$ 1.5. Positive cooperativity has also been observed in the homodimeric mammalian membrane-bound guanylate cyclases. The structure described here provides a reliable model for functional analysis of mammalian guanylate cyclases, which are closely related in sequence.
\end{abstract}

\section{Background}

The second messenger 3',5'-cyclic guanosine monophosphate (cGMP) is central to many signal transduction pathways, primarily eliciting effects by modulating the activities of phosphodiesterases, protein kinases, and ion channels [1-3]. In mammals, cGMP is synthesized by two distinct classes of guanylate cyclases, which are either cytoplasmic or membrane-bound [4]. Both classes of guanylate cyclase share a catalytic module that is closely related in sequence to that of mammalian adenylate cyclases. The catalytic domain is a class III nucleotide cyclase domain [5], which is distributed widely from bacteria to humans. The class III nucleotide cyclase domain is often found fused to diverse regulatory domains, but is also 
found as an isolated protein [6-8]. The mammalian membrane-bound guanylate cyclases, which respond to extracellular peptide binding or to the levels of intracellular $\mathrm{Ca}^{2+}$, function in maintenance of fluid homeostasis, inhibition of myocyte hypertrophy, skeletal development, and visual and olfactory signal transduction [9]. The mammalian soluble guanylate cyclases are regulated primarily by binding of nitric oxide (NO), and they modulate a wide range of physiological functions, such as maintenance of vascular tone, platelet aggregation, and neurotransmission [10]. Dysfunction of guanylate cyclase signaling underlies many pathophysiological conditions, ranging from stroke and hypertension to gastrointestinal disease and neurodegeneration [11-13].

Mammalian soluble guanylate cyclases are heme-containing heterodimers of homologous $\alpha$ and $\beta$ subunits [10]. The N-terminal regulatory domain of each subunit contains a heme-NO and/or oxygen-binding (H-NOX) domain $[14,15]$, and the H-NOX domains of the $\beta$ subunits have been shown to bind the heme cofactor $[16,17]$. The homologous regions of the $\alpha$ subunits do not bind heme, but are predicted to possess a similar fold. The $\alpha$ and $\beta$ subunits each contain a central region, shown to be involved in heterodimerization, that consists of an $\mathrm{H}$ NOXA (H-NOX associated) domain and an amphipathic helical extension predicted to form a coiled-coil $[18,19]$. The catalytic domain is located in the C-terminal segment of the protein, and it associates with the catalytic domain of the partner subunit to form a heterodimeric catalytic unit $[20,21]$. The mechanism of soluble guanylate cyclase activation by NO involves the binding of NO to the heme cofactor [22], but the details of this activation mechanism are unknown. The response of soluble guanylate cyclase to NO is regulated allosterically by nucleotides $[23,24]$, but how this happens is also not understood.

The three-dimensional structure of a guanylate cyclase catalytic domain has not been reported. Crystal structures have been obtained for an oxygen-bound H-NOX domain of a methyl-accepting chemotaxis protein from the obligate anaerobe Thermoanaerobacter tencongensis $[25,26]$ and for NO- and carbon monoxide-bound forms of an $\mathrm{H}-$ NOX domain from a histidine kinase operon in the cyanobacterium Nostoc sp. [27], yielding clues to the mechanism of soluble guanylate cyclase heme ligand recognition and discrimination. Additionally, the crystal structure of the H-NOXA domain of a signal-transduction histidine kinase from Nostoc punctiforme was reported recently [28], revealing that the dimeric H-NOXA domain adopts a Per/Arnt/Sim (PAS) fold and suggesting a mechanism for the preferential heterodimerization exhibited by mammalian soluble guanylate cyclase. Homology modeling based on crystal structures of the related mammalian and bacterial class III adenylate cyclase catalytic domain dimers has provided some information concerning the structure of the catalytic domain of the guanylate cyclases (reviewed in [7]).

There are distinct soluble guanylate cyclases in invertebrates, including insects, nematodes, and algae $[25,29,30]$. Also called atypical soluble guanylate cyclases, several of these have been predicted to function as homodimers instead of heterodimers, and a number have been demonstrated to be regulated by oxygen [31-34]. The core subunit architecture outlined above for the mammalian enzymes is conserved in these atypical ones, with additional domains of unknown function appended to the C-terminus of some of the proteins. Here, we report the structure of the catalytic domain of a soluble guanylate cyclase (CYG12) from the unicellular green algae Chlamydomonas reinhardtii, which shares 40 to 50\% identity with the soluble and membrane-bound guanylate cyclase catalytic domains (Figure 1). The 991-residue fulllength CYG12 protein contains each of the domains present in well-characterized soluble guanylate cyclases as well as an additional C-terminal domain of unknown function, and has the full complement of residues necessary to function as a homodimer. As expected, the C. reinhardtii guanylate cyclase catalytic domain has the same protein fold as the mammalian adenylate cyclases. With minor differences, the positions of the residues necessary for catalysis and nucleotide base recognition are in the same locations for guanylate and adenylate cyclases, although the identities of the base recognition residues are obviously different. Compared to mammalian adenylate cyclase, the crystal structure is in an inactive conformation, with distorted active site structural elements. Based on the structure, we propose a mechanism for the positive cooperativity that is observed for mammalian homodimeric membrane-bound guanylate cyclases and demonstrated by us for the $C$. reinhardtii guanylate cyclase. We speculate that the activation mechanism for the guanylate cyclases involves structural rearrangement analogous to that exhibited by the mammalian adenylate cyclases.

\section{Results and discussion \\ Structure Determination}

We have determined the structure of a $C$. reinhardtii soluble guanylate cyclase catalytic domain dimer by molecular replacement, using the structure of the mammalian adenylate cyclase heterodimer [35] as a search model. The structure contains one catalytic domain dimer per asymmetric unit. During refinement, we observed unexplained peaks in electron density maps around five of the fourteen cysteine residues in the dimer (Cys 499 and Cys 592 of both monomers, and Cys 621 of monomer B). High concentrations of reductant [5-10 mM dithiothreitol and 10 $\mathrm{mM}$ tris(2-carboxyethyl)phosphine] were present during 


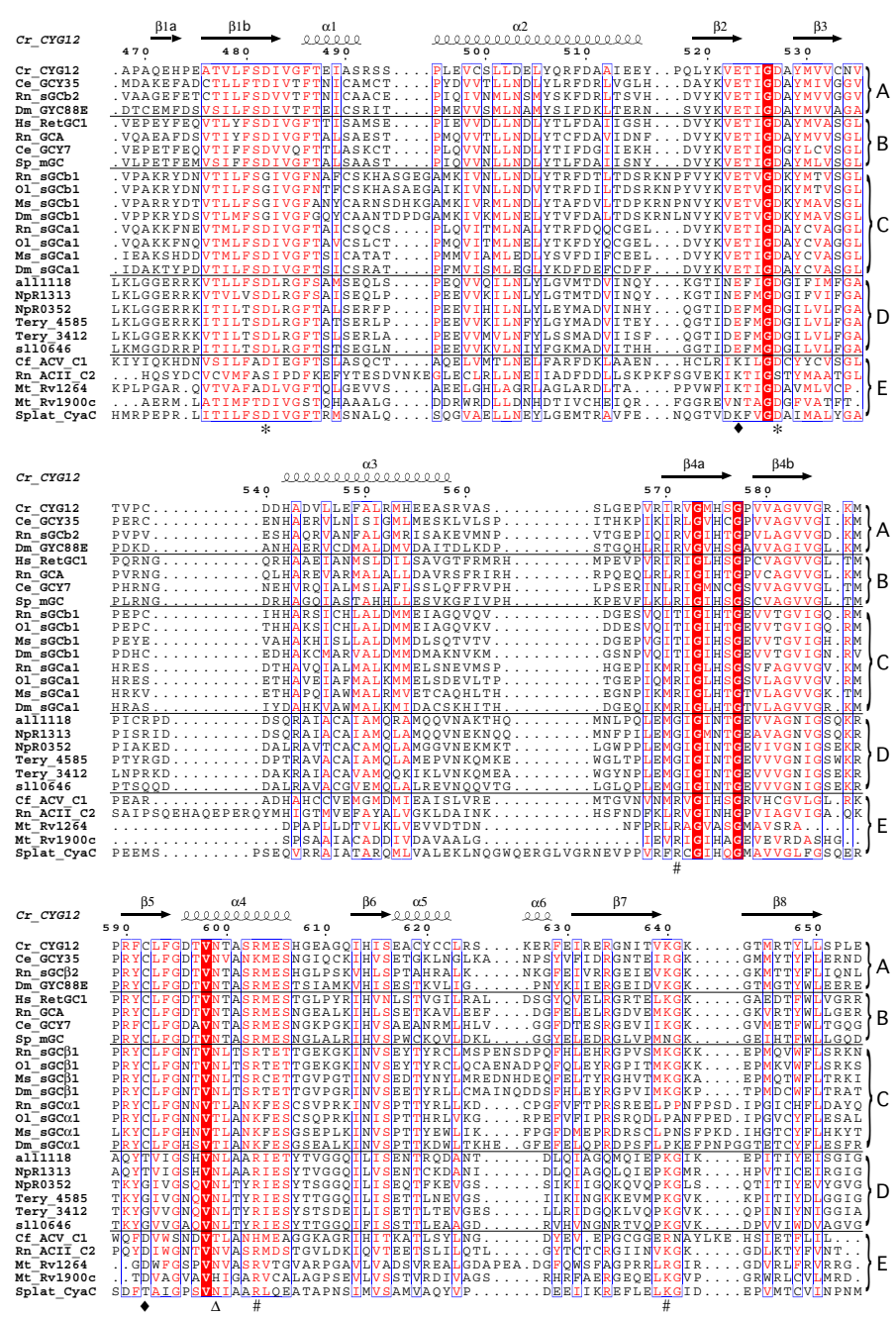

\section{Figure I}

Structural alignment of selected guanylate and adenylate cyclase catalytic domains. Secondary structure annotation and numbering correspond to the guanylate cyclase homolog CYGI 2 from C. reinhardtii. Sequences are grouped as follows: A, atypical soluble guanylate cyclases; B, membrane-bound guanylate cyclases; C, NO-sensing soluble guanylate cyclases; D, putative bacterial guanylate cyclases; E, mammalian and bacterial adenylate cyclases. Functional residues are indicated by symbols: metal binding $(*)$; ribose binding $(\Delta)$; guanine/adenine binding $(\diamond)$; triphosphate binding $(\#)$. Accession numbers are as follows: Chlamydomonas reinhardtii CYGI2 (GenBank XP_001700847), Caenorhabditis elegans GCY35 (GenBank O02298), Rattus norvegicus sGCB2 (GenBank BAB68564), Drosophila melanogaster GYC-88E (GenBank Q8INF0), Homo sapiens RetGCI (GenBank Q02846), R. norvegicus GCA (GenBank PI8910), C. elegans GCY7 (GenBank AAQ6245I), Strongylocentrotus purpuratus mGC (GenBank PI6065), R. norvegicus sGC $\beta$ I (GenBank BAC55087), Oryzias latipes sGC $\beta$ I (GenBank BAA7669I), Manduca sexta sGC $\beta$ I (GenBank AAC6 I264), D. melanogaster sGC $\beta$ I (GenBank), R. norvegicus sGC $\alpha$ I (GenBank AABI7953), O. latipes sGC $\alpha$ I (GenBank BAA76690), M. sexta sGC $\alpha$ I (GenBank AAC6I263), D. melanogaster sGC $\alpha$ I (GenBank AAF56917), Anabaena sp. PCC7I 20 all I I 8 (GenBank NP_485I6I), Nostoc punctiforme PCC73I 02 NpR I 3 I (GenBank YP_00I864972), N. punctiforme PCC73I 02 NpR0352 (GenBank ACC79I35), Trichodesmium erythraeum IMS I0I Tery_4585 (GenBank ABG5356I), T. erythraeum IMSI0I Tery_34I2 (GenBank ABG525 I2), Synechocystis sp. PCC6803 sll0646 (GenBank BAAI6969), Canis familiaris ACV_CI (GenBank ICJU_A), R. norvegicus ACII_C2 (GenBank ICJU_B), Mycobacterium tuberculosis RvI264 (GenBank IYII_A), M. tuberculosis Rv1900c (GenBank IYBU_C), Spirulina platensis CyaC (GenBank IWCI_C). Initial alignments were carried out using the program ClustalX [67]. Sequences were adjusted manually with comparison to results from a structural homology search using the DALI server [45]. Figure I was prepared using the program ESPRIPT [68]. Regions containing residues of $>70 \%$ equivalence (red letters) are boxed with a thin blue line, and absolutely conserved residues are highlighted in red. 
crystallization, making it unlikely that cysteine oxidation or disulfide bond formation are responsible for these features. We wondered whether the apparent modification of the cysteine sidechains might be due to the addition of dimethylarsenic groups via reaction of the cysteine thiol group with the sodium cacodylate [sodium dimethylarsenate, $\left(\mathrm{CH}_{3}\right)_{2} \mathrm{AsO}_{2} \mathrm{Na}$ ] buffer and dithiothreitol reductant present during crystallization [36]. This chemical modification has been observed previously for several proteins crystallized from solutions containing this buffer [37,38]; it has even been used to obtain experimental phases for protein structure solution [39]. Accordingly, we confirmed the presence and location of the dimethylarsenicmodified cysteines by taking advantage of the arsenic anomalous signal to calculate an anomalous difference map, which showed unambiguous peaks of electron density around the dimethylarsenic-modified cysteines (see Additional File 1: Dimethylarsenic cysteine modifications).

The three $\mathrm{C}$-terminal residues of each monomer are disordered, as are residues 564-566 in monomer A and residues 562-565 in monomer $\mathrm{B}$. The final model of the guanylate cyclase dimer includes residues $467-563$ and 567-653 for monomer A and residues 467-561 and 566653 for monomer B. The model also includes 8 phosphate ions and 99 solvent molecules, and was refined to $2.55 \AA$ resolution. After refinement, the final model had working and free R-values [40] of $17.2 \%$ and $21.5 \%$, respectively (Table 1).

\section{The guanylate cyclase fold}

Each guanylate cyclase domain contains a central sevenstranded $\beta$ sheet surrounded by several $\alpha$ helices. Secondary structure elements are named according to the convention for adenylate cyclases $[35,41]$ and are indicated in Figure $2 \mathrm{a}$. The first four $\beta$ strands are part of a $\beta \alpha \beta \beta \alpha \beta$ arrangement of secondary structure elements, a hallmark of the class III nucleotide cyclase fold, several classes of polymerase, and other nucleotidyltransferases [42-44]. Indeed, a search of the structure database with the program DALI [45] identifies the mammalian adenylate cyclase catalytic domains as the closest structural match, followed by several bacterial adenylate cyclases and polymerases. A smaller 3 -stranded $\beta$ sheet, formed by strand $\beta 5$ and extensions of $\beta 1$ and $\beta 4$ ( $\beta 1 \mathrm{a}$ and $\beta 4 \mathrm{~b})$, extends from the core of the domain, and interacts with strands $\beta 2$ and $\beta 3$ of the opposite monomer to form part of the dimer interface. The two monomers in the wreathlike dimer [41] are related by a twofold axis, and a central cleft, formed by the dimer interface, contains the two symmetric active sites (Figure 2b). The two monomers superimpose on each other with a r.m.s. deviation of $0.3 \AA$ for 160 structurally equivalent $C \alpha$ atom pairs. The primary structural differences between the monomers are found in their C-terminal subdomains, particularly in the $\alpha 6-\beta 7$ and $\beta 7-\beta 8$ loops, and are due to differences in crystal packing interactions.

The structure of the guanylate cyclase catalytic domain differs from that of the adenylate cyclases primarily in the elements that connect strands and helices, and in the lessconserved C-terminal subdomains (Figure 1). Biochemical and structural studies have shown that these regions in the adenylate cyclases couple to regulatory proteins such as the heterotrimeric G-protein subunits $G_{s} \alpha, G_{i} \alpha$, and protein kinase $C$ (reviewed in [46]). Differences in sequence and structure between individual catalytic subunits in heterodimeric adenylate and guanylate cyclases also localize to the same regions (Figure 1).

\section{The active site}

The catalytic residues necessary for synthesizing cyclic nucleotides, conserved across all adenylate cyclases and guanylate cyclases (Figure 1), are contributed by structural elements of both monomers at each active site. We will focus on one active site, and refer to each domain as monomer A or B. In the C. reinhardtii guanylate cyclase described here, the catalytic residues are the metal-binding residues Asp 482(A) and Asp 527(A) located in the $\beta 2-\beta 3$ loop and in strand $\beta 1 \mathrm{~b}$, respectively, the ribose-orienting residue Asn 599(B) and the transition-state-stabilizing residue Arg 603(B), both located in helixa4, and the triphosphate-positioning residues $\operatorname{Arg} 571(\mathrm{~A})$ in strand $\beta 4 \mathrm{a}$ and Lys $640(\mathrm{~B})$ in the $\beta 7-\beta 8$ loop (Figure 3a). With the exception of the critical metal-binding residue Asp 527(A) (see below), all of these active site residues are

Table I: Crystallographic data and refinement statistics

\begin{tabular}{ll}
\hline Data collection & \\
\hline Beamline & ALS 8.2.2 \\
Wavelength $(\AA)$ & 1.000 \\
Space group & $\mathrm{P} 3_{2} 21$ \\
Unit cell & $a=123.7, b=123.7, c=62.8$ \\
& $\alpha=90, \beta=90, \gamma=120$ \\
Resolution $(\AA)$ & $28-2.55(2.7-2.55)$ \\
$\mathrm{R}_{\text {merge }}(\%)$ & $7.4(52.1)$ \\
I/ $(\mathrm{I})$ & $7.6(1.3)$ \\
Completeness $(\%)$ & $96.2(98.2)$ \\
Redundancy & $4.3(4.3)$ \\
Refinement & \\
Unique reflections & 32518 \\
Free R test set $(\%)$ & 5 \\
$\mathrm{R}_{\text {work }} / \mathrm{R}_{\text {free }}$ & $17.2 / 21.5$ \\
Monomers per A. U. & 2 \\
No. atoms & 2999 \\
Protein & 2860 \\
Ligand & 40 \\
Solvent & 99 \\
r.m.s. deviation, bond lengths $(\AA)$ & 0.015 \\
r.m.s. deviation, bond angles $(\AA)$ & 1.476 \\
& \\
\hline
\end{tabular}



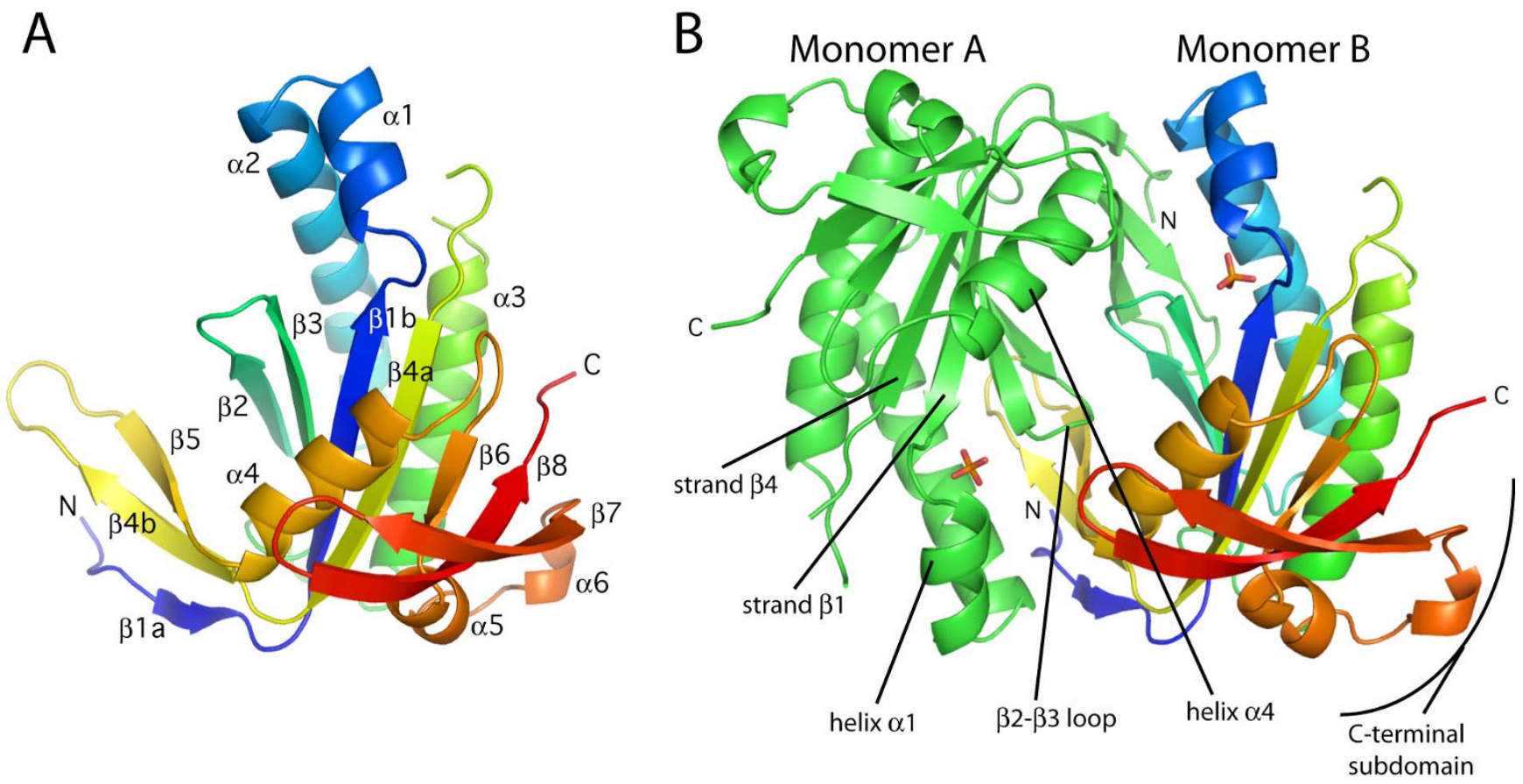

Figure 2

Structural features of the guanylate cyclase domain. A) Structural representation of a guanylate cyclase domain monomer. Elements of secondary structure are labeled according to the nomenclature depicted in Figure I. B) The guanylate cyclase catalytic domain. Monomer $\mathrm{A}$ is colored green, and monomer $\mathrm{B}$ is multi-colored, ranging from blue at the $\mathrm{N}$-terminus to red at the C-terminus. Two of eight phosphate ions are shown and are depicted as stick figures: phosphorus, orange; oxygen, red.

located at positions analogous to their location in the adenylate cyclase active site (Figure 3a); minor conformational differences likely reflect the absence of metals and nucleotides, which would assist in organizing the active site into a catalytically competent conformation.

Modeling based on the adenylate cyclase structures has indicated the mechanism of nucleotide base discrimination $[47,48]$. A glutamic acid and a cysteine, conserved in guanylate cyclases, have been proposed to mediate recognition of the exocyclic amine and carbonyl group of the guanine base, respectively. The specificity-determining residues in mammalian adenylate cyclase, a lysine and an aspartic acid, are located at the same relative positions in the adenylate cyclase protein sequence. In fact, swapping those residues into a guanylate cyclase catalytic domain results in conversion into an adenylate cyclase $[47,48]$, underscoring the equivalence of the catalytic machinery between guanylate and adenylate cyclases. In our structure, the corresponding residues are Glu 523(B) and Cys 592(B), which are situated close to the locations of their adenylate cyclase counterparts (Figure $3 \mathrm{~b}$ ). Local distortions caused by the dimethylarsenic modifications in each monomer appear to prevent optimal sidechain orientation for base recognition. For Cys 592(B), the dimethylarsenic modification of the thiol side chain prevents potential hydrogen bonding interaction with the exocyclic carbonyl group of a substrate GTP molecule (Figure $3 \mathrm{~b}$ ). The modification also results in distortion of the $\beta 2-\beta 3$ loop that contains the metal-binding residue Asp 527(A) and the base-recognition residue Glu 523(A), causing it to adopt a conformation incompatible with binding a metalnucleotide complex (Figure 3a). Together, these local distortions would likely preclude any nucleotide binding in the active site, providing a rationale for our failure to visualize nucleotides and metals that have been soaked into the crystals.

\section{Guanylate cyclase activation}

Activation of the mammalian adenylate cyclase has been proposed to proceed via two steps, based on structures of active and inactive forms of the protein $[35,41,49]$. In the first step, binding of $\mathrm{G}_{\mathrm{s}} \alpha$ between the $\alpha 1-\alpha 2$ and $\alpha 3-\beta 4$ loops of the $\mathrm{C} 2$ subunit causes a $7^{\circ}$ rotation of the core of the $\mathrm{C} 1$ subunit around an axis that runs parallel to the central cleft, priming the active site for catalysis by bringing the catalytic residues from one subunit $\sim 2 \AA$ closer to the catalytic residues of the other [35]. The second step involves the closure of the active site around the bound nucleotide. This closure brings structural elements that bind the metal cofactors and the nucleotide triphosphate moiety, and residues in the opposite subunit that orient the ribose ring and stabilize the transition state, into optimal alignment for catalysis [49] (see Additional file 2: 


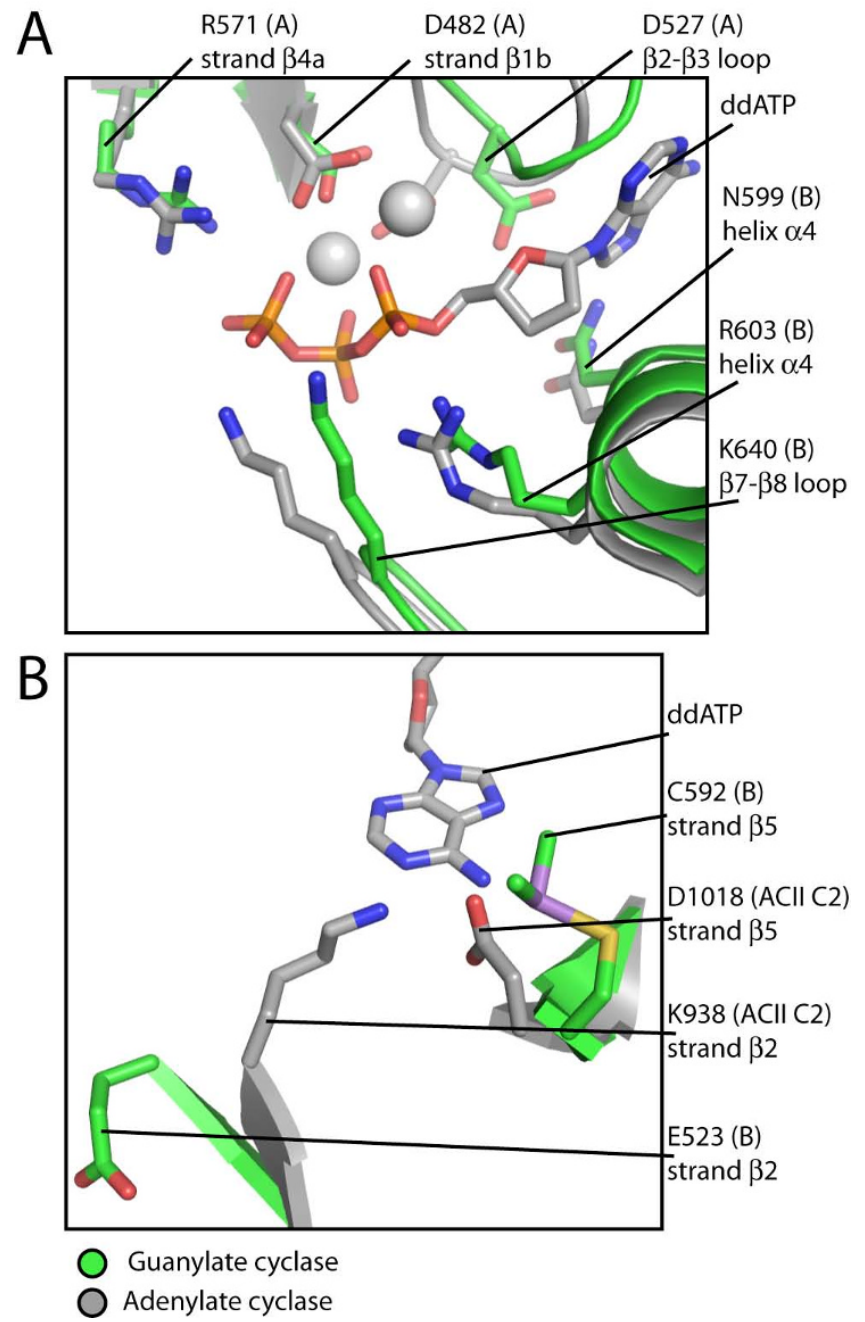

Figure 3

Comparison between guanylate and adenylate cyclase active sites. Monomer B of the guanylate cyclase catalytic domain was superimposed onto the $\mathrm{C} 2$ domain of mammalian adenylate cyclase (PDB ID: ICJU) [49]. Residues and structural elements involved in catalysis and nucleotide recognition are shown. A) Comparison of guanylate and adenylate cyclase catalytic residues. B) Comparison of guanylate and adenylate cyclase base recognition residues. Side chains and structural elements from the guanylate cyclase and adenylate cyclase catalytic domains are colored green and grey, respectively. The nucleotide 2',3'-dideoxyadenosine triphosphate (ddATP) and the two $\mathrm{Mg}^{2+}$ ions are from ICJU. Noncarbon atoms are colored as follows: phosphorus, orange; oxygen, red; nitrogen, blue; sulfur, yellow; arsenic, violet; magnesium, white.

Activation mechanism of mammalian adenylate cyclase). In particular, helix $\alpha 1$ moves towards helix $\alpha 4$ of the opposite subunit, such that a triphosphate interaction site is formed from the helix $\alpha 1$ dipole and a P-loop-like structure between strand $\beta 1 \mathrm{~b}$ and helix $\alpha 1$. The $\mathrm{N}$-terminal end of strand $\beta 4$, the C-terminal end of strand $\beta 1$, and the $\mathrm{N}$ - termini of helices $\alpha 2$ and $\alpha 3$ also shift towards the active site, properly orienting metal-binding and triphosphatebinding residues for catalysis.

Comparison of our guanylate cyclase catalytic domain structure to structures of the mammalian adenylate cyclase suggests that the guanylate cyclase catalytic domain is in an inactive conformation. The dimethylarsenic modifications described above clearly distort several active site residues. But, in addition to these localized changes, the overall orientation of one subunit with respect to the other corresponds to an open state that must close considerably for catalysis to occur, as we discuss further below.

The signature structural change upon activation for all adenylate cyclases is the movement of helix $\alpha 1$ towards the active site and helix $\alpha 4$ in the opposite subunit, regardless of other changes in domain orientations. In our structure, a phosphate ion is bound to the N-terminal end of helix $\alpha 1$ and to the P-loop-like site, suggesting the presence of a likely triphosphate-coordination site. However, when monomer A of the guanylate cyclase structure is superimposed on the active adenylate cyclase $\mathrm{C} 1$ domain, helix $\alpha 1$ has not moved towards the active site, which must occur to properly align all the catalytic residues, bind nucleotide, and achieve an active conformation (Figure 4 ). Instead, the conformation of helix $\alpha 1$ in the guanylate cyclase structure is much closer to that of helix $\alpha 1$ in the inactive adenylate cyclase structure (compare Figure 4 and Additional file 2: Activation mechanism of mammalian adenylate cyclase). Activation of the guanylate cyclase domains in the structure reported here would require the $\mathrm{N}$-terminus of helix $\alpha 1$ of each monomer to move $\sim 3 \AA$ towards helix $\alpha 4$ of the other monomer, resulting in the concomitant shifting of the ends of strands $\beta 1 b$ and $\beta 4 a$ inwards towards the opposite monomer, and leading to an active site configuration similar to that observed for the mammalian adenylate cyclases (Figure 4).

\section{Active site cooperativity}

The mammalian adenylate cyclase catalytic domain heterodimer contains one active site and one catalytically non-functional site - each monomer is missing residues required for catalysis, which are provided by the other monomer. The homodimeric nature of the guanylate cyclase catalytic domain described here suggests that it contains two active sites. In fact, the existence of two active sites has been postulated for mammalian membrane guanylate cyclases, all of which function as homodimers [50]. We sought to confirm this possibility by looking for evidence of cooperativity in activity assays. Activity assays were carried out in the presence of $\mathrm{Mn}^{2+}$, because the activity in the presence of $\mathrm{Mg}^{2+}$ was less than $1 \%$ of that in the presence of $\mathrm{Mn}^{2+}$ (data not shown), as seen for mammalian soluble guanylate cyclase catalytic 
domains [21]. We found that the enzyme exhibits positive cooperativity, with a Hill coefficient of 1.5 (Figure 5), indicating the presence of more than one active site and providing evidence that the active sites interact with each other. Cooperativity has also been observed for other homodimeric cyclases, such as the mammalian membrane-bound guanylate cyclases [51-53], as well as some bacterial adenylate cyclases $[54,55]$. A possible mechanism for communication between the mammalian adenylate cyclase active site and the pseudosymmetric site, where the activator forskolin binds, has been proposed. In the mammalian adenylate cyclase catalytic domain heterodimer, the $\beta 2-\beta 3$ loop of the $\mathrm{C} 1$ monomer, which contains a catalytically essential aspartic acid, is connected via a hydrogen bond and a hydrophobic interaction to the $\beta 2-\beta 3$ loop of the C2 monomer, which forms part of the forskolin binding site. These interactions between the forskolin binding site and the active site provide a possible mechanism by which the binding of forskolin is communicated to the catalytic residues in the active site, resulting in activation [35].
In the absence of nucleotide, it is not clear in our structure how occupation of one active site by nucleotide is detected by the other. The analysis is also complicated by the local distortions near the active site caused by the dimethylarsenic-modified cysteines. However, inspection of our structure provides two possible mechanisms for communication between the active sites. The first mechanism involves a direct connection between residues in the two active sites. The $\beta 2-\beta 3$ loop of each monomer carries both the invariant catalytic residue Asp 527 for one active site and the conserved guanine-binding residue Glu 523 for the opposite active site. We propose that the interaction of either Asp 527 or Glu 523 with nucleotide in one active site could alter the conformation of the loop in which they both reside, resulting in a change in nucleotide affinity or enhanced catalysis in the other active site. The second mechanism involves propagation of local changes in one active site, through changes in elements of secondary structure, to the other active site. As noted above, upon substrate binding, helix $\alpha 1$ and strands $\beta 1$ and $\beta 4$ in monomer A move inward towards crucial residues in helix $\alpha 4$

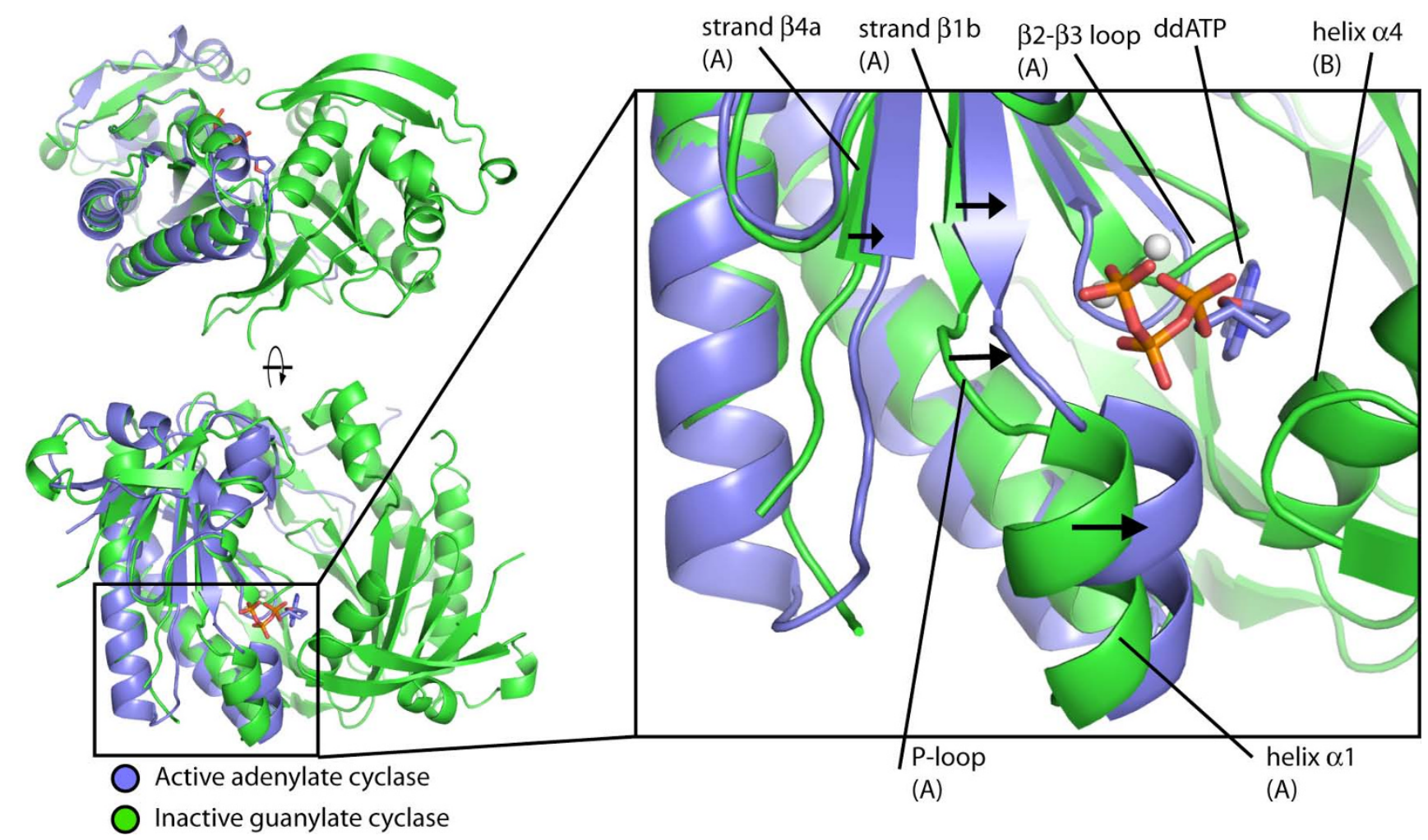

\section{Figure 4}

Proposed guanylate cyclase activation mechanism. Comparison of helix $\alpha \mathrm{I}$ in the guanylate cyclase structure with helix $\alpha$ l of the active mammalian adenylate cyclase structure indicates that the guanylate cyclase structure is in an inactive state. Monomer $B$ of the guanylate cyclase structure was superimposed onto the $C 2$ domain of the active adenylate cyclase structure (PDB ID: ICJU) [49] and monomer A was superimposed onto the $\mathrm{CI}$ domain of the same adenylate cyclase structure. The C2 domain is omitted for clarity. The $\mathrm{Cl}$ domain of the active adenylate cyclase structure is colored blue, and the guanylate cyclase structure is colored green. The nucleotide 2',3'-dideoxyadenosine triphosphate (ddATP) and $\mathrm{Mg}^{2+}$ ions from ICJU are shown as a stick figure and spheres: phosphorus, orange; oxygen, red; nitrogen, blue; magnesium, white. 
of monomer $\mathrm{B}$, bringing residues from both monomers into correct alignment for catalysis in one active site (Figure 4). This movement also brings helix $\alpha 4$ of monomer A, which lies directly above, and packs against, strands $\beta 1$ and $\beta 4$ of monomer $\mathrm{A}$, towards monomer $\mathrm{B}$. As helix $\alpha 4$ of monomer A carries residues necessary for catalysis at the other active site, this movement might allow nucleotide binding at one active site to effectively begin preorganizing the other active site for nucleotide binding.

\section{Interaction with regulators}

The activating conformational transition of helix $\alpha 1$ and attendant shift in adjacent $\beta$ strands might be facilitated by a domain rotation like that observed for the mammalian adenylate cyclase $\mathrm{C} 1$ monomer upon $\mathrm{G}_{\mathrm{s}} \alpha$ binding [35]. In the adenylate cyclases, the $\alpha 1-\alpha 2$ and $\alpha 3-\beta 4$ loops of the $\mathrm{C} 2$ domain form a groove into which the switch II helix of $G_{s} \alpha$ is docked. The docking of $G_{s} \alpha$ brings

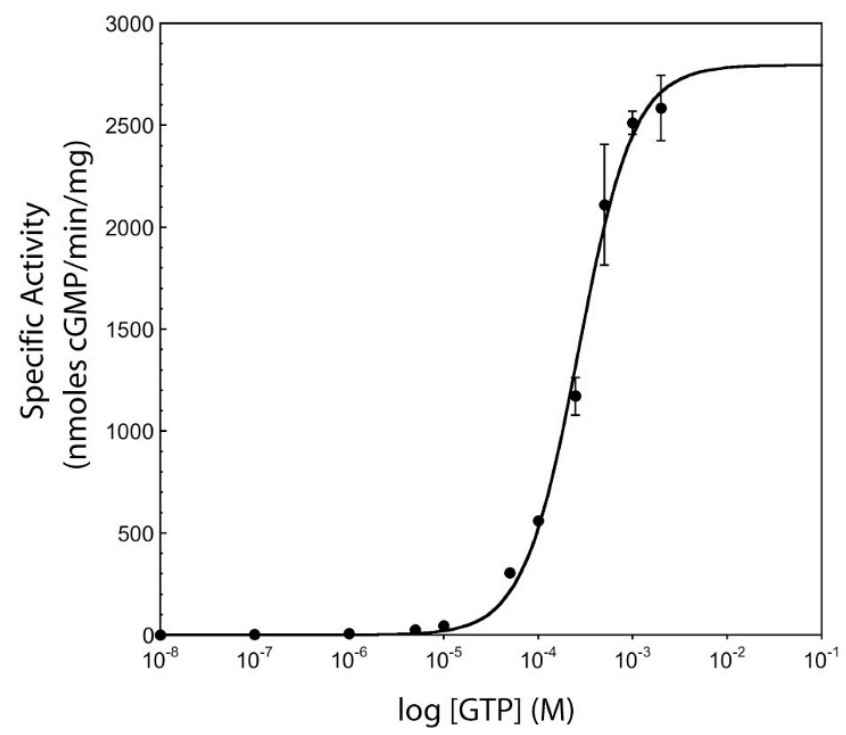

Figure 5

Communication between active sites. Plot of guanylate cyclase activity at increasing concentrations of substrate

GTP. Guanylate cyclase ( $5 \mu \mathrm{g}$ ) was incubated for 2 min at 24

${ }^{\circ} \mathrm{C}$ with the indicated concentrations of GTP in the presence of $4 \mathrm{mM} \mathrm{MnCl} 2$ and cGMP was measured. Data were fit to

the equation $\frac{V_{\max }(S)^{n}}{\left(S_{0.5}\right)^{n}+S^{n}}$, where $V_{\max }$ is the maximum activity, $S$ is the concentration of GTP, $S_{0.5}$ is the substrate concentration at which half-maximal velocity is reached, and $n$ is the Hill coefficient. From the fit, $V_{\max }=2795 \pm 117$ nmoles

$\mathrm{cGMP} / \mathrm{min} / \mathrm{mg}, \mathrm{S}_{0.5}=269 \pm 26 \mu \mathrm{M}$, and $n=1.49 \pm 0.16$. A Hill coefficient greater than $\mathrm{I}$ indicates the presence of interacting active sites. about the rotation of the $\mathrm{C} 1$ domain. A similar groove is found in the analogous location on the guanylate cyclase dimer structure (Figure 6). It is tempting to speculate that some regulatory element, such as a soluble guanylate cyclase H-NOX sensor domain, might interact with this region in an analogous fashion, altering the balance of conformations in the catalytic domain. It is also possible that interaction of regulators with entirely different structural elements, such as the C-terminal subdomains, may be required to activate the guanylate cyclase catalytic domain. Answers to such questions await the structure of an active guanylate cyclase domain in the presence of regulatory elements.

\section{Conclusion}

We report the first structure of a eukaryotic guanylate cyclase catalytic domain. The resemblance of the domain to that of the mammalian adenylate cyclase is unsurprising, given the sequence and functional similarity between them. Nevertheless, more than ten years have elapsed between the first reports of the structures of the adenylate cyclases [35,41] and our results, presented here. The difficulty in crystallizing a guanylate cyclase domain may reflect an increased intrinsic flexibility in the guanylate cyclase domain relative to the adenylate cyclase domain, and it is possible that we succeeded in part because of the fortuitous cysteine modifications that may have increased the rigidity of the domain, facilitating crystallization. We have been unable as yet to crystallize the catalytic domain in the absence of these modifications.

The high degree of sequence conservation between the soluble guanylate cyclase catalytic domain described here and the catalytic domains of mammalian soluble and membrane-bound guanylate cyclases ( 40 to $50 \%$ identity) suggests that our structure will serve as a superior model for functional studies, compared to the mammalian adenylate cyclase catalytic domains (25 to $30 \%$ sequence identity). Our structure indicates that the differences between the adenylate and guanylate cyclase are generally localized to flexible regions, some of which are proposed to mediate coupling with regulatory domains and other control elements. While specific differences in regulatory interactions are likely determined by the sequence and local structure of these variable elements, the overall activation mechanism, involving conformational switching by helix $\alpha 1$ and attendant changes in the adjacent $\beta$ sheet, is expected to be conserved.

\section{Methods}

\section{Cloning and protein purification}

PCR was used to amplify the gene encoding a 991-residue soluble guanylate cyclase homolog CYG12 (GenBank: XM_001700795) from a C. reinhardtii cDNA library obtained from the Chlamydomonas Center [56]. Forward 
and reverse PCR primers were 5'-ATGCTGGGCTGGTATGACCGT-3' and 5'-TTACTCCAAACACGGGTTGTCA-3', respectively. PCR products were phosphorylated, bluntcloned into the vector pGEM, and verified by sequencing (UC Berkeley DNA Sequencing Facility). The guanylate cyclase catalytic domain was expressed and purified using a SUMO-based system (LifeSensors) as follows: residues 468-655, which comprise the catalytic domain of CYG12, were subcloned into a vector containing the yeast SUMO homolog SMT3 with an N-terminal His-tag, and the fusion protein was expressed in Escherichia coli Tuner(DE3) (Invitrogen) for $18 \mathrm{~h}$ at $20^{\circ} \mathrm{C}$. The fusion protein was purified from supernatant by passage over a HisTrap Ni Sepharose affinity column (GE Healthcare). A His-tagged version of the SMT3-specific protease was used to cleave the N-terminal SMT3 fusion partner from the guanylate cyclase domain, which was separated from the protease and SMT3 by a second Ni affinity step. The guanylate cyclase domain was further purified by Q Sepharose anion-exchange chromatography, followed by gel filtration into a final buffer of $25 \mathrm{mM}$ triethanolamine, pH 7.5, $25 \mathrm{mM} \mathrm{NaCl}$, and $10 \mathrm{mM}$ dithiothreitol. Purified protein was concentrated and stored at $-20^{\circ} \mathrm{C}$ until use. Protein concentrations were determined by absorbance using the calculated extinction coefficient $\varepsilon_{280}=7680 \mathrm{M}^{-1}$ $\mathrm{cm}^{-1}$.

\section{Crystallization and $X$-ray data collection}

Crystals were grown using the sitting-drop vapor diffusion method. Equal volumes $(200 \mathrm{nl})$ of protein [40-60 mg/ $\mathrm{ml}$ in $25 \mathrm{mM}$ triethanolamine, $\mathrm{pH} 7.5,25 \mathrm{mM} \mathrm{NaCl}, 5-$ $10 \mathrm{mM}$ dithiothreitol, $10 \mathrm{mM}$ tris(2-carboxyethyl)phosphine] were mixed with crystallization solution $[0.1 \mathrm{M}$ sodium cacodylate, $\mathrm{pH}$ 5.0-6.4, 42-62\% saturated $\left(\mathrm{NH}_{4}\right)_{2} \mathrm{HPO}_{4} \mathrm{~J}$ and then equilibrated with a $100-\mu \mathrm{l}$ reservoir of the same crystallization buffer at $20^{\circ} \mathrm{C}$. Crystals grew in the trigonal space group and appeared within 1-2 days. Crystals were transferred to a solution of mother liquor containing $28 \%$ glycerol as a cryoprotectant, and cryocooled and stored in liquid nitrogen. Diffraction data were collected at $100 \mathrm{~K}$ using synchrotron radiation at beam line 8.2.2 at the Advanced Light Source, Lawrence Berkeley National Laboratory. Reflections were integrated and scaled with the programs MOSFLM [57] and SCALA [58]. The structure was solved by molecular replacement with the program PHASER [59] using the mammalian adenylate cyclase catalytic domain (PDB code 1AZS) [35] as the search model, and the space group was identified as $\mathrm{P}_{2} 2$ 21. Map improvement was carried out using ARP/ wARP [60] and RESOLVE [61]. The model was built using COOT [62] and refined using PHENIX [63]. Six TLS domains were used during refinement: residues 467-475 and 578-595, monomer A/B; residues 476-560, 569577, and 596-608, monomer A/B; and residues 613-651, monomer A/B. Analysis of model quality was carried out

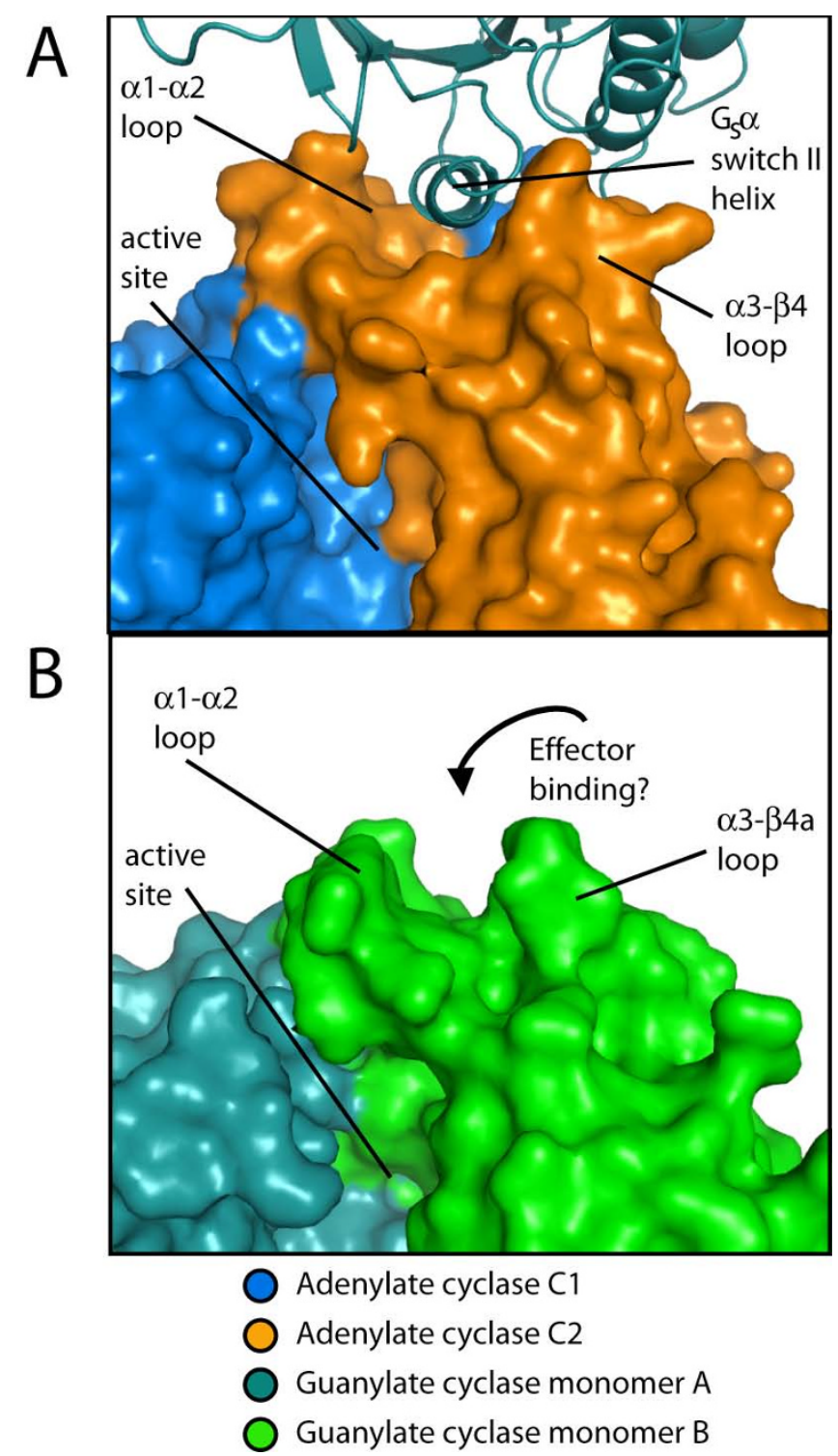

Figure 6

A potential binding site for a regulatory control element. A) Structure of the mammalian adenylate cyclase catalytic domain bound to the activator $\mathrm{G}_{s} \alpha$ (PDB ID ICJU)

[49]. The switch II helix of $G_{s} \alpha$ binds in a groove on the $C 2$ domain between the $\alpha \mathrm{I}-\alpha 2$ and $\alpha 3-\beta 4$ a loops, priming the catalytic domain for nucleotide binding. A surface representation of the adenylate cyclase catalytic domain is shown, and $\mathrm{G}_{s} \alpha$ is shown as a ribbon cartoon. The $\mathrm{Cl}$ domain is colored blue, the $C 2$ domain is colored orange, and $G_{s} \alpha$ is colored teal. B) Surface representation of the guanylate cyclase catalytic domain in the same orientation as the adenylate cyclase domain in $\mathrm{A}$. A groove similar to that used by adenylate cyclase to bind to $G_{s} \alpha$ is located between the $\alpha I-\alpha 2$ and $\alpha 3$ $\beta 4 \mathrm{a}$ loops, and may serve as a site for interaction of control elements with the guanylate cyclase catalytic domain. Monomer $A$ is colored teal, and monomer $B$ is colored green. 
using MOLPROBITY [64]. Figures were prepared using PYMOL [65]. The atomic coordinates and structure factors have been deposited in the Protein Data Bank (3ET6).

\section{Guanylate cyclase assays}

Guanylate cyclase assays were performed in duplicate at $24^{\circ} \mathrm{C}$ as described previously [66]. Assays contained $5 \mu \mathrm{g}$ of guanylate cyclase in $25 \mathrm{mM}$ triethanolamine, $\mathrm{pH} 7.5$, $25 \mathrm{mM} \mathrm{NaCl}, 4 \mathrm{mM} \mathrm{MnCl}{ }_{2}$ or $\mathrm{MgCl}_{2}$ and $5 \mathrm{mM}$ dithiothreitol. Assays were initiated by addition of indicated amounts of GTP, and were quenched after 2 minutes by addition of $400 \mu \mathrm{l}$ of $125 \mathrm{mM} \mathrm{Zn}\left(\mathrm{CH}_{3} \mathrm{CO}_{2}\right)_{2}$ and $500 \mu \mathrm{l}$ of $125 \mathrm{mM} \mathrm{Na}_{2} \mathrm{CO}_{3}$. cGMP was quantified using a cGMP enzyme immunoassay kit, format $\mathrm{B}$ (Biomol), per the manufacturer's instructions. Experiments were repeated three times to ensure reproducibility.

\section{Authors' contributions}

JAW designed and performed research, analyzed data, and drafted the manuscript. ERD performed research and analyzed data. MHL analyzed data. MAM designed research and analyzed data. JK designed research, analyzed data, and drafted the manuscript. All authors read and approved the final manuscript.

\section{Additional material}

\section{Additional file 1}

Dimethylarsenic cysteine modifications. Five cysteine residues are modified through a reaction with the sodium cacodylate and dithiothreitol in the crystallization buffer, resulting in addition of dimethylarsenic to the cysteine thiols. Experimental anomalous difference density contoured at 5 $\sigma$ is shown superimposed onto the refined guanylate cyclase structure. Sidechains are show as stick figures and colored as follows: carbon, green; sulfur, yellow; arsenic, violet.

Click here for file

[http://www.biomedcentral.com/content/supplementary/14726807-8-42-S1.png]

\section{Additional file 2}

Activation mechanism of mammalian adenylate cyclase. Helix $\alpha 1$ in the C1 domain of mammalian adenylate cyclase undergoes a significant conformational change going from an inactive structure to an active structure. A hypothetical inactive structure of the mammalian adenylate cyclase catalytic domain was generated as previously described [35]: the C2 domain structure from 1AZS was superimposed on chain A from the structure of a C2 domain homodimer (PDB ID: 1AB8) [41], and the C1 domain structure from $1 \mathrm{AZS}$ was superimposed on chain $B$ from $1 \mathrm{AB} 8$. The nucleotide- and $G_{s} \alpha$-bound active C1/C2 dimer structure 1CJU [49] was then superimposed onto the $\mathrm{C} 2$ domain of the inactive model. The inactive model is colored white, and the active structure is colored blue. $G_{s} \alpha$ and the C2 domain of 1 CJU are omitted for clarity. The nucleotide 2',3'-dideoxyadenosine triphosphate (ddATP) and $\mathrm{Mg}^{2+}$ ions from $1 \mathrm{CJU}$ are shown as a stick figure and spheres: phosphorus, orange; oxygen, red; nitrogen, blue; magnesium, white.

Click here for file

[http://www.biomedcentral.com/content/supplementary/14726807-8-42-S2.png]

\section{Acknowledgements}

We would like to thank Carmen Schwechheimer, Caleb Cassidy-Amstutz, and Bianca Lee for technical assistance, and Nicholas Levinson and Markus Seeliger for critical reading of the manuscript. This work was supported in part by NIH grant GM077365 (M.A.M.). J.A.W. was supported by an American Heart Association Postdoctoral Fellowship. The Advanced Light Source is supported by the U.S. Department of Energy under Contract DEAC03-76SF00098 at the Lawrence Berkeley National Laboratory.

\section{References}

I. Kaupp UB, Seifert R: Cyclic nucleotide-gated ion channels. Physiol Rev 2002, 82(3):769-824.

2. Munzel T, Feil R, Mulsch A, Lohmann SM, Hofmann F, Walter U: Physiology and pathophysiology of vascular signaling controlled by guanosine $3^{\prime}, 5^{\prime}$-cyclic monophosphate-dependent protein kinase. Circulation 2003, I08(18):2|72-2183.

3. Rybalkin SD, Yan C, Bornfeldt KE, Beavo JA: Cyclic GMP phosphodiesterases and regulation of smooth muscle function. Circ Res 2003, 93(4):280-29I.

4. Padayatti PS, Pattanaik P, Ma X, Akker F van den: Structural insights into the regulation and the activation mechanism of mammalian guanylyl cyclases. Pharmacol Therapeut 2004, 104(2):83-99.

5. Linder JU, Schultz JE: The class III adenylyl cyclases: multi-purpose signalling modules. Cell Signal 2003, I5(12): 108I-1089.

6. Linder JU: Substrate selection by class III adenylyl cyclases and guanylyl cyclases. IUBMB Life 2005, 57( I 2):797-803.

7. Linder JU: Class III adenylyl cyclases: molecular mechanisms of catalysis and regulation. Cell Mol Life Sci 2006, 63(15):|736-|75|.

8. Wu J, Bai J, Bao Q, Zhao F: Lineage-specific domain fusion in the evolution of purine nucleotide cyclases in cyanobacteria. J Mol Evol 2008, 67(I):85-94.

9. Kuhn M: Structure, regulation, and function of mammalian membrane guanylyl cyclase receptors, with a focus on guanylyl cyclase-A. Circ Res 2003, 93(8):700-709.

10. Denninger JW, Marletta MA: Guanylate cyclase and the $\bullet \mathbf{N O}$ cGMP signaling pathway. Biochim Biophys Acta 1999, I4I I(23):334-350

II. Takahashi T: Pathophysiological significance of neuronal nitric oxide synthase in the gastrointestinal tract. J Gastroenterol 2003, 38(5):42I-430.

12. Bredt DS: Endogenous nitric oxide synthesis: biological functions and pathophysiology. Free Radical Res 1999, 3 I (6):577-596.

13. Dawson VL, Dawson TM: Nitric oxide in neurodegeneration. Prog Brain Res 1998, I I 8:21 5-229.

14. Karow DS, Pan D, Davis JH, Behrends S, Mathies RA, Marletta MA: Characterization of functional heme domains from soluble guanylate cyclase. Biochemistry 2005, 44(49): 16266-16274.

15. lyer LM, Anantharaman V, Aravind L: Ancient conserved domains shared by animal soluble guanylyl cyclases and bacterial signaling proteins. BMC Genomics 2003, 4(I):5.

16. Zhao Y, Marletta MA: Localization of the heme binding region in soluble guanylate cyclase. Biochemistry 1997, 36(50):15959-15964.

17. Wedel B, Humbert P, Harteneck C, Foerster J, Malkewitz J, Böhme E, Schultz G, Koesling D: Mutation of His- 105 in the beta I subunit yields a nitric oxide-insensitive form of soluble guanylyl cyclase. P Natl Acad Sci USA 1994, 9 I (7):2592-2596.

18. Koglin M, Behrends S: A functional domain of the alphal subunit of soluble guanylyl cyclase is necessary for activation of the enzyme by nitric oxide and YC-I but is not involved in heme binding. J Biol Chem 2003, 278(14): | 2590-I2597.

19. Zhou Z, Gross S, Roussos C, Meurer S, Muller-Esterl W, Papapetropoulos A: Structural and functional characterization of the dimerization region of soluble guanylyl cyclase. J Biol Chem 2004, 279(24):24935-24943.

20. Wedel B, Harteneck C, Foerster J, Friebe A, Schultz G, Koesling D: Functional domains of soluble guanylyl cyclase. J Biol Chem 1995, 270(42):2487I-24875.

21. Winger JA, Marletta MA: Expression and characterization of the catalytic domains of soluble guanylate cyclase: Interaction with the heme domain. Biochemistry 2005, 44(10):4083-4090. 
22. Ignarro LI: Heme-dependent activation of soluble guanylate cyclase by nitric oxide: regulation of enzyme activity by porphyrins and metalloporphyrins. Semin Hematol 1989, 26(I):63-76.

23. Chang FJ, Lemme S, Sun Q, Sunahara RK, Beuve A: NO-dependent allosteric inhibitory role of a second-nucleotide binding site in soluble guanylyl cyclase. I Biol Chem 2005, 280(I2): II513-11519.

24. Ruiz-Stewart I, Tiyyagura SR, Lin JE, Kazerounian S, Pitari GM, Schulz S, Martin E, Murad F, Waldman SA: Guanylyl cyclase is an ATP sensor coupling nitric oxide signaling to cell metabolism. $P$ Natl Acad Sci USA 2004, I 0 I(I):37-42.

25. Nioche P, Berka V, Vipond J, Minton N, Tsai AL, Raman CS: Femtomolar sensitivity of a NO sensor from Clostridium botulinum. Science 2004, 306(570 I): I550-I553.

26. Pellicena P, Karow DS, Boon EM, Marletta MA, Kuriyan J: Crystal structure of an oxygen binding heme domain related to soluble guanylate cyclases. P Natl Acad Sci USA 2004, I0I(35): I 2854-I2859.

27. Ma X, Sayed N, Beuve A, Akker F van den: NO and CO differentially activate soluble guanylyl cyclase via a heme pivot-bend mechanism. EMBO J 2007, 26(2):578-588.

28. Ma X, Sayed N, Baskaran P, Beuve A, Akker F van den: PAS-mediated dimerization of soluble guanylyl cyclase revealed by signal transduction histidine kinase domain crystal structure. J Biol Chem 2008, 283(2): | |67-I I78.

29. Morton DB: Invertebrates yield a plethora of atypical guanylyl cyclases. Mol Neurobiol 2004, 29(2):97-I I6.

30. Merchant SS, Prochnik SE, Vallon O, Harris EH, Karpowicz SJ, Witman GB, Terry A, Salamov A, Fritz-Laylin LK, Marechal-Drouard L, et al.: The Chlamydomonas genome reveals the evolution of key animal and plant functions. Science 2007, 3 I 8(5848):245-250.

31. Cheung BH, Cohen M, Rogers C, Albayram O, de Bono M: Experience-dependent modulation of $C$. elegans behavior by ambient oxygen. Curr Biol 2005, I 5(I 0):905-917.

32. Gray JM, Karow DS, Lu H, Chang AJ, Chang JS, Ellis RE, Marletta MA Bargmann $\mathrm{Cl}$ : Oxygen sensation and social feeding mediated by a C. elegans guanylate cyclase homologue. Nature 2004, 430(6997):317-322.

33. Vermehren A, Langlais KK, Morton DB: Oxygen-sensitive guanylyl cyclases in insects and their potential roles in oxygen detection and in feeding behavoirs. J Insect Physiol 2006, 52(4):340-348.

34. Huang SH, Rio DC, Marletta MA: Ligand binding and inhibition of an oxygen-sensitive soluble guanylate cyclase, Gyc-88E, from Drosophila. Biochemistry 2007, 46(5I): $15115-15122$.

35. Tesmer J], Sunahara RK, Gilman AG, Sprang SR: Crystal structure of the catalytic domains of adenylyl cyclase in a complex with $\mathbf{G}_{\mathrm{s}} \alpha . \mathbf{G T P} \gamma$ S. Science 1997, 278(5345): $1907-1916$.

36. Scott N, Hatlelid KM, MacKenzie NE, Carter DE: Reactions of arsenic(III) and arsenic(V) species with glutathione. Chem Res Toxicol 1993, 6(I): 102-106

37. Goldgur $Y$, Dyda F, Hickman AB, Jenkins TM, Craigie R, Davies DR: Three new structures of the core domain of HIV-I integrase: an active site that binds magnesium. P Natl Acad Sci USA 1998, 95(16):9150-9154.

38. Raman CS, Li H, Martásek P, Král V, Masters BS, Poulos TL: Crystal structure of constitutive endothelial nitric oxide synthase: a paradigm for pterin function involving a novel metal center. Cell 1998, 95(7):939-950.

39. Greenwald J, Le V, Butler SL, Bushman FD, Choe S: The mobility of an HIV-I integrase active site loop is correlated with catalytic activity. Biochemistry 1999, 38(28):8892-8898.

40. Brunger AT: Free $\mathbf{R}$ value: a novel statistical quantity for assessing the accuracy of crystal structures. Nature 1992, 355(6359):472-475.

4I. Zhang G, Liu Y, Ruoho AE, Hurley JH: Structure of the adenylyl cyclase catalytic core. Nature 1997, 386(6622):247-253.

42. Artymiuk PJ, Poirrette AR, Rice DW, Willett P: A polymerase palm in adenylyl cyclase? Nature 1997, 388(6637):33-34.

43. Murzin AG: How far divergent evolution goes in proteins. Curr Opin Struc Biol 1998, 8(3):380-387.

44. Pei J, Grishin NV: GGDEF domain is homologous to adenylyl cyclase. Proteins 200I, 42(2):210-216.
45. Holm L, Sander C: Mapping the protein universe. Science 1996 , 273(5275):595-602.

46. Hurley JH: Structure, mechanism, and regulation of mammalian adenylyl cyclase. J Biol Chem 1999, 274( I 2):7599-7602.

47. Sunahara RK, Beuve A, Tesmer JJ, Sprang SR, Garbers DL, Gilman AG: Exchange of substrate and inhibitor specificities between adenylyl and guanylyl cyclases. I Biol Chem 1998, 273(26): 16332-16338.

48. Tucker CL, Hurley JH, Miller TR, Hurley JB: Two amino acid substitutions convert a guanylyl cyclase, RetGC-I, into an adenylyl cyclase. P Natl Acad Sci USA I 998, 95(I I ):5993-5997.

49. Tesmer J], Sunahara RK, Johnson RA, Gosselin G, Gilman AG, Sprang SR: Two-metal-Ion catalysis in adenylyl cyclase. Science 1999, 285(5428):756-760.

50. Liu Y, Ruoho AE, Rao VD, Hurley JH: Catalytic mechanism of the adenylyl and guanylyl cyclases: modeling and mutational analysis. P Natl Acad Sci USA 1997, 94(25): I34 |4-I34|9.

5I. Gazzano H, Wu HI, Waldman SA: Activation of particulate guanylate cyclase by Escherichia coli heat-stable enterotoxin is regulated by adenine nucleotides. Infect Immun I991, 59(4): 1552-1557.

52. Ivanova K, Heim JM, Gerzer R: Kinetic characterization of atrial natriuretic factor-sensitive particulate guanylate cyclase. Eur J Pharmacol 1990, 189(4-5):317-326.

53. Chrisman TD, Garbers DL, Parks MA, Hardman JG: Characterization of particulate and soluble guanylate cyclases from rat lung. J Biol Chem 1975, 250(2):374-38I

54. Shenoy AR, Srinivasan N, Subramaniam M, Visweswariah SS: Mutational analysis of the Mycobacterium tuberculosis Rv1625c adenylyl cyclase: residues that confer nucleotide specificity contribute to dimerization. FEBS Lett 2003, 545(2-3):253-259.

55. Sinha SC, Wetterer M, Sprang SR, Schultz JE, Linder JU: Origin of asymmetry in adenylyl cyclases: structures of Mycobacterium tuberculosis Rv I 900c. EMBO / 2005, 24(4):663-673.

56. The Chlamydomonas Center [http://www.chlamy.org/]

57. Leslie AGW: Recent changes to the MOSFLM package for processing film and image plate data. In Joint CCP4 + ESF-EAMCB Newsletter on Protein Crystallography Volume 26. Edited by: Warrington LD. UK; 1992.

58. The CCP4 suite: programs for protein crystallography. Acto Crystallogr D 1994, 50(Pt 5):760-763.

59. McCoy AJ, Grosse-Kunstleve RW, Storoni LC, Read RJ: Likelihoodenhanced fast translation functions. Acta Crystallogr D 2005, 61:458-464

60. Perrakis A, Morris R, Lamzin VS: Automated protein model building combined with iterative structure refinement. Nat Struct Biol 1999, 6(5):458-463.

61. Terwilliger TC: Maximum-likelihood density modification. Acta Crystallogr D 2000, 56(Pt 8):965-972.

62. Emsley P, Cowtan K: Coot: model-building tools for molecular graphics. Acta Crystallogr D 2004, 60:2। 26-2। 32

63. Adams PD, Grosse-Kunstleve RW, Hung LW, loerger TR, McCoy AJ, Moriarty NW, Read RJ, Sacchettini JC, Sauter NK, Terwilliger TC: PHENIX: building new software for automated crystallographic structure determination. Acta Crystallogr D 2002, 58:1948-1954.

64. Davis IW, Leaver-Fay A, Chen VB, Block JN, Kapral GJ, Wang X, Murray LW, Arendall WB 3rd, Snoeyink J, Richardson JS, et al.: MolProbity: all-atom contacts and structure validation for proteins and nucleic acids. Nucleic Acids Res 2007:W375-383.

65. The PyMOL Molecular Graphics System [http:// www.pymol.org]

66. Winger JA, Derbyshire ER, Marletta MA: Dissociation of nitric oxide from soluble guanylate cyclase and heme-nitric oxide/ oxygen binding domain constructs. J Biol Chem 2007, 282(2):897-907.

67. Larkin MA, Blackshields G, Brown NP, Chenna R, McGettigan PA Mcwilliam H, Valentin F, Wallace IM, Wilm A, Lopez R, et al.: Clustal $W$ and Clustal $X$ version 2.0. Bioinformatics 2007 23(2I):2947-2948

68. Gouet P, Courcelle E, Stuart DI, Metoz F: ESPript: analysis of multiple sequence alignments in PostScript. Bioinformatics 1999 , I5(4):305-308. 